\title{
Analysing COVID-19 Verified, Recuperate and Death Cases in Ethiopia Using ARIMA Models
}

\author{
Birhanu Betela Warssamo
}

Department of Statistics, College of Natural and Computational Sciences, Hawassa University, Hawassa, Ethiopia, P. O. Box: 05

\section{KEYWORDS:}

COVID-19 Cases;

Days;

Autoregressive

Moving Average

\begin{abstract}
Applying a successful prediction of the confirmed, recovered and deaths is thought to be the basic requirement to successfully control the spreading rate of diseases. Time series models have extensively been considered as the suitable methods to forecast the Integrated confirmed, recovered and deaths because of the virus. Aim of this research is to apply the Autoregressive Integrated Moving Average (ARIMA) modelling approach for projecting COVID-19 confirmed, recovered and deaths cases in Ethiopia. Over strict follows of all phase of Box-Jenkins strategy, ARIMA $(1,1,1)$, ARIMA $(16,1,2)$ and ARIMA $(0,1,1)$ models for confirmed case, recovered and death case, respectively were selected as the best models for predicting corona virus cases of Ethiopia. Using these models, a forecasts of five month a heads future situation of COVID-19 confirmed case, recovered and death case (Jan 3, 2021 to May 3, 2021) has made. The results showed that in the coming five months from Jan 3, 2020 to May 3, 2021, the number of COVID-19 confirmed, recovered and deaths cases in Ethiopia may reach up to 320,597 ; 168,912 and 4438, respectively. Generally, the size of the corona virus distribution was increased from time to time in the past ten month, until $3^{\text {rd }}$ Jan, 2021, and it is expected to continue quicker than before for the coming 5-months, until the end of May, 2021, in Ethiopia and more rapidly than before while the peak will remain unknown yet. Therefore, an effective implementation of the preventive measures and a rigorous compliance by avoiding negligence with the rules such as prohibiting public gatherings, travel restrictions, personal protection measures, and social distancing may improve the spreading rates of the virus. Further, through updating more new data with continuous reconsideration of predictive model provide useful and more precise prediction
\end{abstract}

\section{INTRODUCTION}

The primary event of corona virus (COVID-19) eruption was informed on December 31, 2019 in Wuhan and then, it has been put across the universal deadly disease owning harsh new type of hazard to individual vigour and life in a continues way. According to World Health Organization (2020), more than 135,045 COVID-19 cases, 2,083 deaths, and 121,594 recoveries have been reported in Ethiopia and 101,483,628 COCID-19 cases, 2,185,413 deaths and $73,386,329$ recoveries and 25,908,918 active cases among these $25,801,597(99.6 \%$ ) in 
mild condition and $110,289(0.4 \%)$ series or critical cases and 73,386,329 (97\%) are recovered and 2,185,413(3\%) are dead across the globe during the study period (WHO, 2020). The quantity of COVID-19 cases rapidly goes up in mid-January, and the infection quickly blowout beyond China's boundaries. As of this research, it has extended to 219 countries all over the globe (WHO, 2019). Despite broadening COVID-19 influences, there are key public health questions. How many individuals will be diseased? How does the condition vary day by day? How many individuals will die? These inquiries could be re-joined by predicting the possible futures of this contagion via time series models. However, broadly used time series models and tools do not essentially have high forecasting precision, particularly for medical researches (Huppert and Katriel, 2013). By fitting models and creating precision forecasting unfailingly may improve community health judgment-making (Yonar et al., 2020).

On March 13, 2020, the Federal Ministry of Health has confirmed a corona virus disease (COVID-19) case in Addis Ababa, Ethiopia. The case, which was announced on the 13th of March 2020, is the first one to be reported in Ethiopia since the beginning of the outbreak in China in December 2019. The case is a 48- year old Japanese man reported to have travelled from Japan to Burkina Faso and then arrived in Ethiopia. He developed symptoms and presented at the health centre in Addis Ababa from where the rapid response team (RRT) moved him to the isolation facility in YekaKotebe. He was clinically stable, with no serious symptoms. Subsequently, on 16 March 2020, the administration of Ethiopia have been announcing to appliance numerous defensive actions such as emerging community alertness about over all behaviour of the infection and its stoppage tools like cleansing of ways and marketplaces, isolation of mistrusted and diseased cases, lockdown of the universities, schools, house of worship, and announcing state of crisis. Ethiopian administration has applied protective measures directly after the infection has been stated the world disease. Yet, the spread has continued quick for the reason that supreme of the individuals have been carelessness in certain of defensive actions such as public distancing and mass assembly. On the other, populace movement through the border from the next-door nations like Sudan, Djibouti, Kenya and Somalia has a lion part for the current movement of quick spread of infection in Ethiopia. Accordingly, COVID-19 spread would get thoughtful in Ethiopia, and its coming movement is also likely to depend directly or indirectly on the spread of COVID-19 inside and outside of all those neighboring African countries.

Investigating the trends and predicting the coming indices of the infection in Ethiopia must be accompanied in order to put in a place operative monitoring policies. Though the spread mechanisms of the infection is not totally recognized, the number of new cases are harshly growing, and the effects of inhibition should be assessed on numerical data where numerical investigation is extra significant.

Actually, several states in the world up to now have functional data-driven statistical models alike Autoregressive Integrated Moving Average (ARIMA) for forecasting of future movements of the communicable infection. Subsequently, to model the exponential growing rate of the virus, Yichi et al. (2020) and Gupta and Pal (2020) conducted their study using a 
linear based time series model, Auto Regressive Moving Average (ARIMA family) model. Time series forecasting models like ARIMA is the ordinary method which gives clothed forecasts on time series data in fast time (Hyndman et al, 2008). This method of investigation has been extensively functional, for its consistency and rapid application by numerous stakeholders. Hence the aim of this research is to put on the Autoregressive Integrated Moving Average (ARIMA) modelling approach for predicting corona virus (COVID-19) confirmed cases, recovered and deaths in Ethiopia to empower the community health organizations to conduct trust worthy day-to-day prediction and to arise with appropriate intervention policies, deliver direct and long-term route of the illness and contribute to the body of knowledge in epidemiologic study method.

\section{MATERIALS AND METHODS}

\section{Study Area}

The research was conducted in Ethiopia, where the virus quickly spread and disturbing. According to the UN population estimates, whole population of the country is $114,963,836$ peoples.

\section{Research data}

The everyday time series data of COVID-19 of Ethiopia from 13 March 2020 to January 3, 2021 were collected from the official website of Johns Hopkins university (2020): https://data.humdata.org/dataset/ethiopiacovid19-cases. SPSS Version 20 statistical software was functional to achieve statistical data investigation on the confirmed, recovered and deaths case of COVID-19 datasets.

\section{Technique of data study}

Time series analysis aims to tell consistent and expressive statistics and use this information to forecast upcoming values of the series. Time series models try to predict the upcoming values by investigating the former and present. It contemplates past data and tries to arise some procedure which will clarify those existences and forecast upcoming values. The distinct feature of time series analysis is consecutive observations are typically dependent and that the investigation must take methods to classify the designs which characteristically happen in the data. Based on this detail, amongst time series methods, this research applied ARIMA model in order to evaluate the upcoming movement situation of COVID-19 in Ethiopia.

\section{Auto Regressive Integrated Moving Average (ARIMA) modelling}

It is the other most regularly used time series models as it takes into account varying movements, periodic fluctuations and random disturbances in the time series. It also appropriate for all types of data, containing nonstationary data, which is if there is no systematic change in mean (no trend), no systematic change in variance and periodic variations has removed (Box and Jenkins, 1976). In practice, most of the time series are non-stationary and Wei, 2006 commends eliminating any nonstationary sources of variation in time series data. In most case, common method for attaining stationary is to put on consistent differencing and $\log$ transformation to the original time series $\left(\mathrm{x}_{\mathrm{t}}\right)$. If differencing a series $\mathrm{d}$ times makes it into a stationary and then series said to follows an autoregressive integrated 
moving average process, denoted by ARIMA ( $p$, d, q) and can be written as:

$\varphi(\beta) \nabla^{d} X_{t}=\theta(\beta) W_{t}$

Where:

Autoregressive operator can be expressed as: $\varphi(\beta)=1-\varphi \beta-\varphi_{2} \beta^{2}-\ldots-\varphi_{p} \beta^{p}$

Moving average operator

$\theta(\beta)=1+\theta_{1} \beta+\ldots+\theta_{p} \beta$

Differencing operator $\left(\nabla^{d}\right)=(1-\beta)^{d}$ it is the expression of $\mathrm{d}^{\text {th }}$ consecutive differencing so as to make series stationary. $\mathrm{W}_{\mathrm{t}}$ is a Gaussian white noise series with mean zero and variance $\left(\delta_{w}{ }^{2}\right)$.

Testing for stationary: Before emerging a Box-Jenkins modelling process, it is significant to check whether the data under study encounters basic suppositions such as series stationary. A time series is well-thought-out as stationary if its statistical properties such as mean and variance are constant over time (Box and Jenkins, 1976). Many testing procedures for stationary are planned in the literature. In this research, correlogram test were functional for testing whether the series is stationary.

The correlogram test: It is one way to characterize a series with respect to its dependence over time. It usually recognized as sample autocorrelation function (ACF), which is plot of sample ACF coefficient against observation difference in time (lag). As indicated in (Box and Jenkins, 1976) if the sample ACF decays very slowly in non-seasonal and seasonal lag snit indicates that differencing is wanted and an insinuation for series nonstationary.

Constructing ARIMA model: As planned by (Granger and Newbold, 1986) method, in order to construct ARIMA model for a specific time series data, must follow four phases: Model identification, estimation of model parameters, Diagnostic checking for the identified model, Application of the model (forecasting).

Model Identification: With this phase, the amount of differencing compulsory attaining stationary and the order of both the seasonal and non-seasonal AR and MA operators are determined. The autocorrelations function (ACF) and the partial autocorrelation functions (PACF) are the two most valuable tools in any attempt at time series model identification (Shumway and Stoffer, 2010). To determine the number of differencing (d), non-seasonal autoregressive (p) and moving average (q) parameters, the guideline specified in (Lehmann and Rode, 2001) are used. Therefore, if PACF Cuts off after lags $\mathrm{q}$ and ACF tail off, then $\operatorname{ARIMA}(0, \mathrm{~d}, \mathrm{q})$ model is identified. If ACF cut off after lag $\mathrm{p}$ and PACF tail off, ARIMA (p, d, 0 ) model is obtained. Finally, if both ACF and PACF tail off, then the recognized model will be ARIMA (p, d, q).

Parameter Estimation: After selecting the most suitable ARIMA model, the parameters are estimated by Maximum Likelihood Estimation.

Diagnostic Checking: it deals with the residual assumptions in order to decide whether the residuals from fitted model are independent, constant variance(Lehmann and Rode, 2001). 
Visual Analysis: It is a method for investigating plot of the residual over time. If visual reviews of the plot tell that they are randomly distributed over time, then it is a residual independence (Lehmann and Rode, 2001).

\section{Residual Autocorrelation Function (RACF):}

With this test, to say that residual follows a white noise process, roughly $95 \%$ of the autocorrelation coefficient should fall within the range of $\pm 1.96 / \mathrm{squ}(\mathrm{n})$ (Grasselli et al, 2020).

If the nominated model is insufficient, the three-step model building process with other model is typically repeated several times until a satisfactory model obtained. The final selected model can then be used for forecast determinations (Box and Jenkins, 1976).

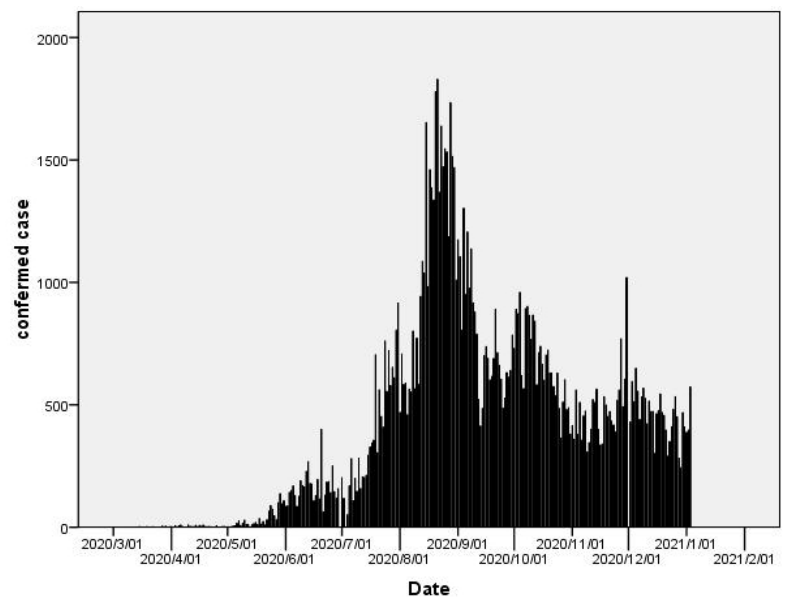

Figure 1 Distribution of confirmed cases of covid-19 in Ethiopia(Mar 3, 2020- Jan 3, 2021)

Predicting: It is last phase in time series modelling, the aim is to forecast future values of a time series, $X t+m, m=1,2, \ldots$ based on the data collected to the present, $X=\{X t, X t-$ $1, \ldots, X l\}$.

$X_{t+m}=\phi_{1} X_{t+m-1}+\ldots+\phi_{p+d} X_{t+m-p-d}-\theta_{1} W_{t+m-1}-\ldots-\theta_{q} W_{t+m-q}$

\section{RESULTS}

\section{Descriptive analysis results}

The first confirmed case of the COVID-19 in Ethiopia (the index case) is described on 13 March 2020 with one infected foreigner. Then after, the administration of Ethiopia has without delay in use different measures to put off and manage the infection, but the case has been expontially increasing and widely distributing until Sep 1, 2020. From Aug 01, 2020 to Oct 01, 2020 large number of people are infected by the virus and the number of infection shows slight decline until the study period Jan 3, 2021 (Fig.1). Similarly, the first recovered cases reported on Jun 01, 2020 and the recovery cases increased parallel with the confirmed cases. There was a large number recovery cases during December 1, 2020 to January 1, 2021 (Fig 2).

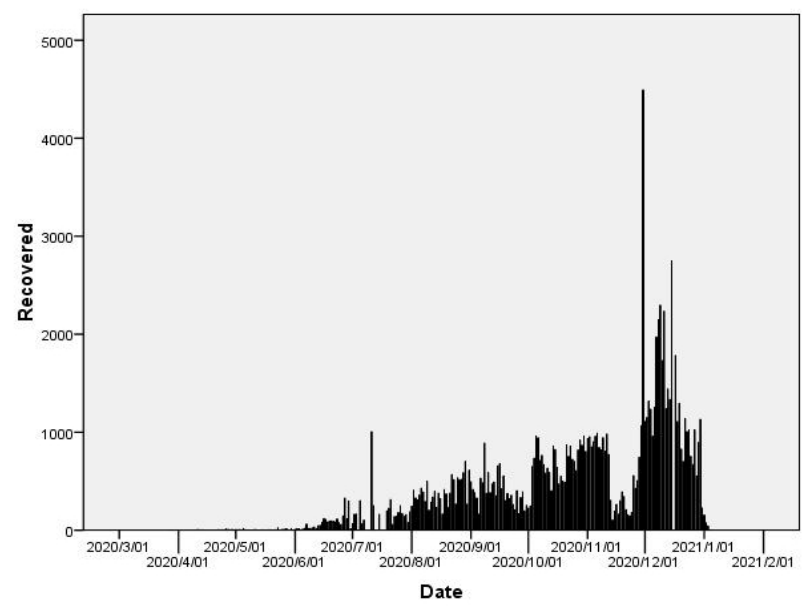

Figure2: Distribution of covid-19 recovered case in Ethiopia (13 Mar, 2020-Jan 3, 2021)

As shown in Fig 3, the first mortality/death case was reported on April 2, 2020. The number of deaths was increasing with the increase of the number of confirmed cases. High number of deaths in Ethiopia was reported during Aug 03, 2020 - Oct 1, 2020. As shown on Fig 4, the 
confirmed numbers of cases are increasing and the recovery case is also more increasing parallel to the confirmed cases.

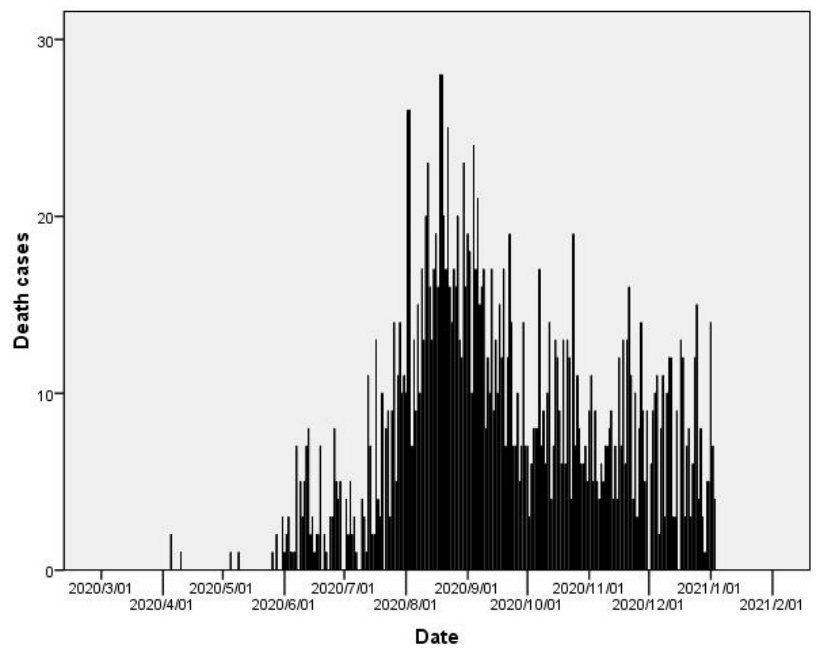

Figure 3: Distribution of death cases in Ethiopia (Mar 3, 2020-Jan3, 2021)

The number of death cases is also increasing but interestingly the recovery cases (green line) is very closed to confirmed cases (blue line) but

\section{Results from ARIMA modelling of COVID- 19 case}

Result in testing stationary: The first step in every time series investigation containing ARIMA modelling is to see whether the time series is stationary. In this research, time series plot and Autocorrelation Function (ACF) were practical to check the stationary. The pattern of the series of COVID-19 confirmed cases, recovery and death cases shows increasing trend indicating the mean and the variance of the the death (yellow line) is going away from the confirmed cases (blue line), that is, the death cases are not increasing in the same level as confirmed cases and the death line is getting flat.

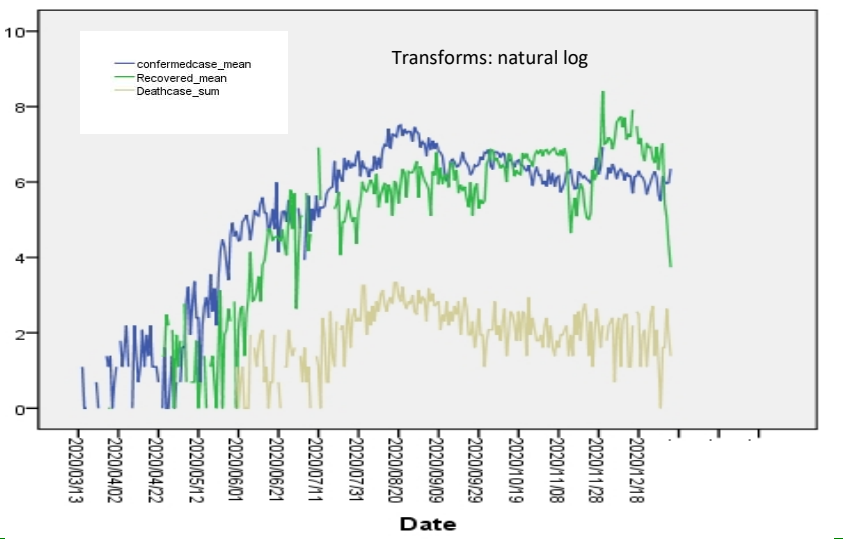

Figure 4: Covid-19 confirmed, recovered and death cases in Ethiopia (Mar 3, 2020-Jan3, 2021).

series are not constant throughout time and this shows the process is not stationary and the data need to be differencing and with the one step differencing, $\mathrm{d}=1$, we got stationary data.

\section{Result in model identification for confirmed cases:}

From Table 1 ARIMA $(1,1,1)$ and ARIMA $(5,1,1)$ are recommended since they have lest Bayesian Information Criterion (BIC) and highest adjusted R-square, but by B-J methodology, parsimonious models give better 
forecast than over-parameterized models.

Therefore, ARIMA $(1,1,1)$ is selected.

Table 1: Tentative models

\begin{tabular}{lllll}
\hline Confirmed cases & ARIMA(1,1,1) & ARIMA(1,1,2) & ARIMA(5,1,1) & ARIMA(5,1,2) \\
\hline Adj R square & 88.9 & 88.9 & 89.3 & 89.3 \\
BIC & 9.855 & 9.877 & 9.911 & 9.933 \\
\hline
\end{tabular}

Result in model identification for recovered case:

From Table2 ARIMA $(15,1,1)$ and $\operatorname{ARIMA}(1,1,2)$ are selected but by B-J methodology , ARIMA $(1,1,2)$ is the best model to forecast the confirmed cases. But residual
$\mathrm{ACF}$ and PACF are not flat, that is lags 16 and 15 are very significant indicating there is white noise in the series and we should re estimate the model by adding AR(16) in the model. Thus the appropriate model to forecast recovery case is ARIMA $(16,1,2)$.

Table 2: Tentative models

\begin{tabular}{lllll}
\hline Recovered cases & ARIMA(1,1,1) & ARIMA(1,1,2) & ARIMA(15,1,1) & ARIMA(15,1,2) \\
\hline Adjusted R square & 60.4 & 60.6 & 66.1 & 66.4 \\
BIC & 11.614 & 11.631 & 11.775 & 11.788 \\
\hline
\end{tabular}

\section{Result in model identification for death case:}

From auto and partial auto correlation plot a candidate MA(q) models were recommended. That is ARIMA $(0,1,1)$ models is recommended.

Result in model diagnosis: According to (Box and Jenkins, 1976), all the models that accepted and satisfied all residual tests and the parameters meaningfully vary from zero must be included and nominated as candidate model for forecast. Residuals ACF and partial ACF plot for confirmed, recovered and death cases, all the lags are inside the $95 \%$ confidence interval showing there is no problem of white nose so the models are ready for use of prediction. 
Table 3: ARIMA models \& coefficients for best selected model.

\begin{tabular}{|c|c|c|c|c|c|c|}
\hline COVID-19 cases & Model & BIC & R-squared & MAPE & Coefficient & P value \\
\hline \multirow[t]{2}{*}{ Confirmed case } & ARIMA $(1,1,1)$ & 9.855 & 889 & 0.309 & $\hat{\phi}_{1=-0.233}$ & 0.016 \\
\hline & & & & & $\hat{\theta}=0.417$ & 0.00 \\
\hline \multirow[t]{6}{*}{ Recovered case } & ARIMA(16,1,2) & 11.732 & 0.69 & 0.439 & $\hat{\phi}_{1}=0.257$ & 0.00 \\
\hline & & & & & $\hat{\phi}_{2}=0.230$ & 0.00 \\
\hline & & & & & $\hat{\phi}_{3}=0.129$ & 0.034 \\
\hline & & & & & $\hat{\phi}_{6}=0.185$ & 0.003 \\
\hline & & & & & $\hat{\phi}_{15}=-0.330$ & 0.00 \\
\hline & & & & & $\hat{\theta}=0.998$ & 0.00 \\
\hline Deaths cases & $\operatorname{ARIMA}(0,1,1)$ & 2.649 & 0.679 & 0.407 & $\hat{\theta}=0.801$ & 0.00 \\
\hline
\end{tabular}

\section{Predicting precision assessment}

If the fitted models achieve well in predicting, the estimate error will be comparatively minor and the Mean Absolute Percentage Error (MAPE) should be close to 5\%. From Table 3, it can be observed that the accuracy of forecasts measured by the Mean Absolute Percentage
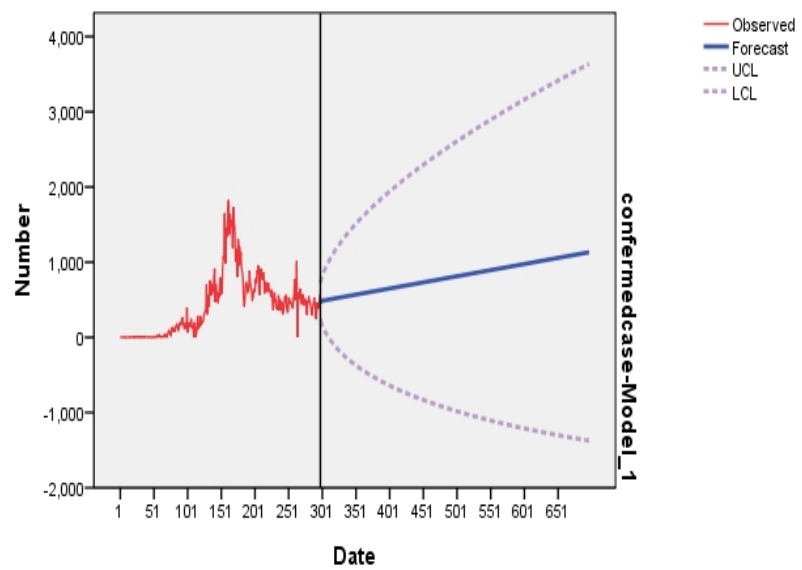

Figure 5: Forecasted value with $95 \%$ prediction interval for confirmed cases in Ethiopia
Error (MAPE) cast out to be 3.09\% for ARIMA $(1,1,1)$ model, $4.39 \%$ for ARIMA $(16,1,2)$ and $4.07 \%$ for ARIMA $(0,1,1)$ model, which are relatively less than $5 \%$. This implies that those models would perform well in predicting the confirmed, recovery and death cases of COVID-19 well.

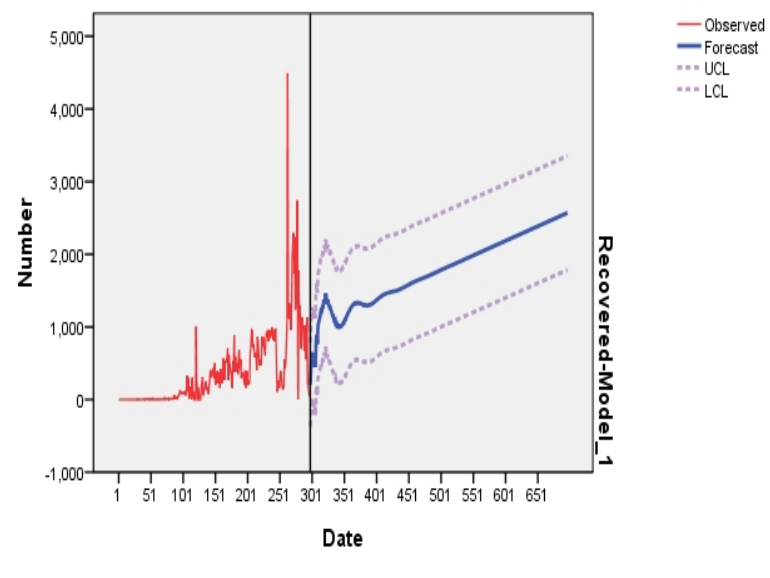

Figure 6: Forecasted value with $95 \%$ prediction interval for recovered cases in Ethiopia 
Fig 5, Fig 6 and Fig 7 presents prediction of COVID-19 confirmed, recovered and death cases and it gives the direction and trend of the epidemic and predicts the likely development of future epidemics. The Figs shows, a forecasts of five month a heads future situation of COVID19 confirmed case, recovered and death case
(Jan 3, 2021 to May 3, 2021). Infected cases, recovered and death cases are expected to considerably increase in the coming five months. By the end of May 3, 2021, cumulative confirmed, recovered and death cases across Ethiopia, will reach $320,597,168,912$ and 4438 respectively.

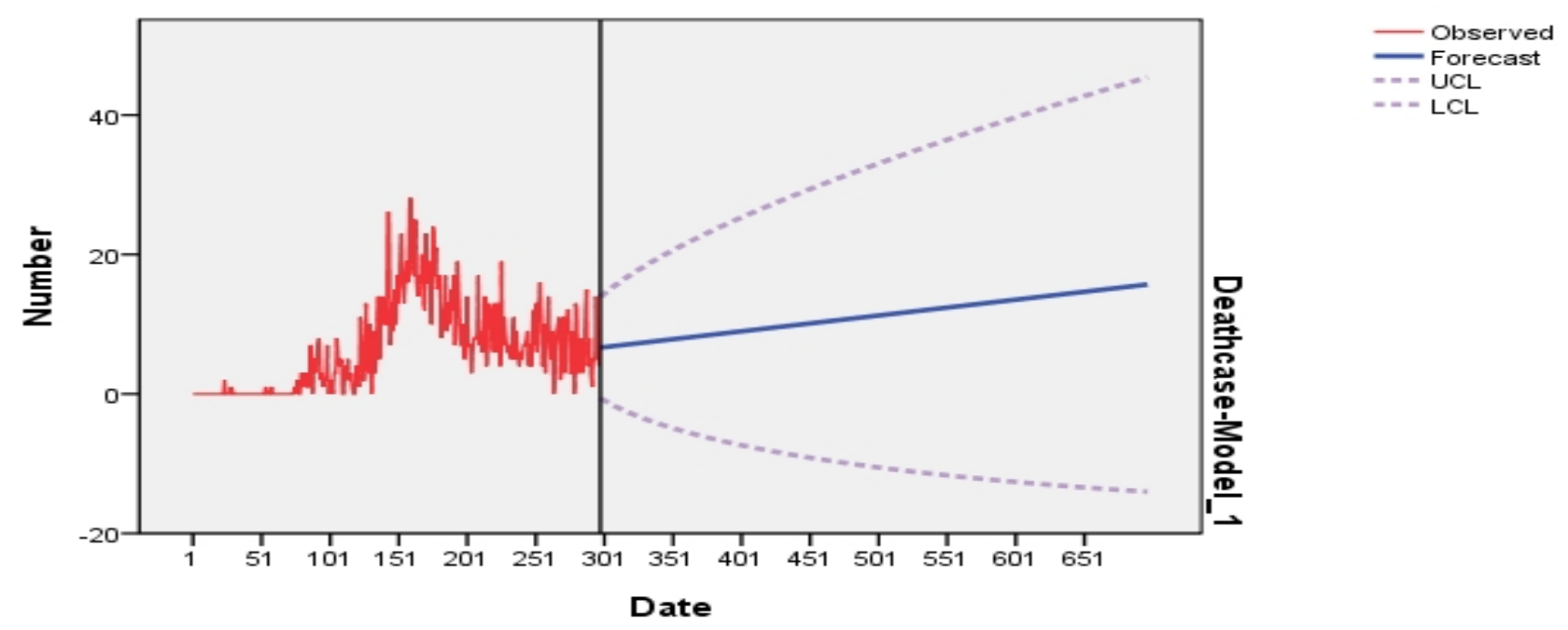

Figure 7 Forecasting value with $95 \%$ prediction interval for deaths case in Ethiopia

\section{DISCUSSION}

It is crucial to generate consistent and appropriate forecasting model that can assistance health system administrations and other stakeholders to prevent the extra blowout of COVID-19. Time series forecasting models is the statistical method which provides a clothed forecast and has been extensively practical for trend of communicable sickness in rapid time (Fanelli and Piazza 2020). ARIMA models are predictive technique that offers a good forecast and has been widely used for the rapid trend of infectious diseases (Holt, 1957, Hyndman et al, 2008, Shumway and Stoffer, 2010).
Over strict follows of all phase of Box-Jenkins strategy, ARIMA $(1,1,1)$, ARIMA $(16,1,2)$ and ARIMA $(0,1,1)$ models for confirmed case, recovered and death case respectively were selected as the best models for predicting COVID-19 cases of Ethiopia. Time series analysis of COVID-19 provides the direction and movement of the epidemic and forecasts the probable progress of upcoming epidemics. Using these models, a forecasts of five month a heads future situation of COVID-19 confirmed case, recovered and death case (Jan 3, 2021 to May 3, 2021) has made. In diverse area, a lot of numeric researches have been showed to forecast new case of COVID-19 case using ARIMA model (Gupta and Pal,2020, Li et al., 
2020, Yonar et al., 2020 and Zhan et al., 2020) which are consistent with this research. In line with the current study, Li et al. (2020) and Das (2020) also used ARIMA model to predicting COVID-19 epidemic in India and China, respectively and showed that the infected case were increasing.

The number of reported cases in the country is expected to exceed 160,585 in the five months of 2021. At the end of May, inline projected cumulative infections across Ethiopia to hit 156,610 on average (Hyndman et al, 2008), while the real value until 19 May was just 189,137 cases and 1352 deaths (Yonar et al., 2020). This means that the number of people who are corona-virus-positive in Ethiopia can increase more than predicted (Hyndman et al, 2008). However, in this analysis, the cumulative confirmed, cumulative recovered and cumulative death forecast cases on May 3, 2021, are 320,597, 168,912 and 4438, respectively. Similar to the predicted values in this study, variation of pandemics in Ethiopia in the first five months has been shown to increase (Hyndman et al, 2008). The scholars disprove the effect of geographical difference and temperature in reducing the distribution of COVID-19 (Granger and Newbold, 1986). However, Covid-19 has caused serious global social and economic distress. Thus, COVID-19, regulated only by pre-protective strategies. However, no aspect is evaluated in this study due to the unavailability of exposure data.

\section{CONCLUSIONS}

Since the study shows the increase in the number of deaths and confirmed cases in Ethiopia, more attention should be given to the control and prevention of Covid-19. Unless the
Government of Ethiopia applies a mechanism to control the infection, the condition is going to be worse and affect the country further. The study therefore suggested that control mechanisms of the pandemic should be implemented on a permanent root. Since ARIMA model is an effort to predict the future forecast of the distribution of COVID-19, based on current data, so that the institutions have to prepare policies.

\section{References}

Box G. and Jenkins G. 1976. Time series analysis: Forecasting and control. Holden day.

Canters for Disease Control and Prevention. Corona virus Disease 2019 (COVID-2019). [https://www. cdc.gov/coronavirus/2019-ncov]. Accessed on 1-282021.

Das R. 2020. Forecasting incidences of COVID-19 using Box-Jenkins method for the period July 12Septembert 11, 2020: A study on highly affected countries. Chaos, Solitons and Fractals 140: 110248. Link: https://bit.ly/2MBS4XR

Domenico B., Marta G., Lazzaro V., Silvia A., and, Massimo C. 2020. Application of the ARIMA model on the COVID- 2019 epidemic data set. Data Brief 29: 105340 .

Fanelli D. and Piazza F. 2020. Analysis and forecast of COVID-19 spreading in China, Italy and France. Chaos, Solitons\& Fractals, 134, 1e12https://doi.org/10.1016/j.chaos.2020.109761

Fattorini D. and Regoli F. 2020. Role of the chronic air pollution levels in the Covid-19 outbreak risk in Italy. Environ Pollu 264: 114732.

Granger K. and Newbold J. 1986. Forecasting economic time series. USA: Academic Press.

Grasselli G., Pesenti A. and Cecconi M. 2020. Critical care utilization for the COVID-19 outbreak in lombardy, Italy: Early experience and forecast during an emergency response. J. Am. Med. Asso. 323(16): 1545-1546.

Gupta R and Pal S.K.. 2020. Trend analysis and forecasting of COVID-19 outbreak in India. MedRxiv. https://doi.org/10.1101/2020.03.26.2004451.

Holt CE. 1957. Forecasting seasonal and trends by exponentially weighted averages (O.N.R. Memorandum No. 52). Carnegie Institute of Technology, Pittsburgh USA. https://doi.org/10.1016/j.ijforecast. 
Huppert A and Katriel G. 2013. Mathematical modelling and prediction in infectious disease epidemiology. ClinMicrobiol Infect. 19(11):999-1005. https://doi.org/10.1111/1469-0691.12308 PMID: 24266045

Hyndman R.J., Koehler A.B., Ord J.K., and Snyder R.D. 2008. Forecasting with Exponential Smoothing: The State Space Approach. Berlin Germany: Springer, $372 \mathrm{p}$.

Khana F. and Gupta R. 2020. ARIMA and NAR based prediction model for time series analysis of COVID19 cases in India. Journal of Safety Science and Resilience 1: 12-18. Link: https://bit.ly/3ot9YtC

Lehmann A. and Rode M. 2001. Long-term behaviour and cross-Correlation Water quality analysis of the river elbe, Germany. Water Res. 35: 2153-2160.

Li Q., Feng W. and Quan Y. 2020. Trend and forecasting of the COVID-19 outbreak in China. J Infect 80 (4): 469-496. https://doi.org/10.1016/j.jinf.2020.02.014

Papastefanopoulos V., Linardatos P., and, Kotsiantis S. 2020. A Comparison of Time Series Methods to Forecast Percentage of Active Cases per Population. Applied sciences 10: $3880 . \quad$ Link: https://bit.ly/35bxKTy

Shumway R. and Stoffer D. 2010. Time series analysis and its applications with $\mathrm{R}$ Examples ( $3^{\text {rd }}$ ed.). Springer

Wei W. 2006. Time series analysis univariate and multivariate (p. 478). New York-USA: AddisonWesley Publishing Company, Inc.
WHO. Corona virus disease 2019 (COVID-19) situation reports. [https://www.who.int/emergencies/ diseases/novel-coronavirus-2019/situation-reports] Accessed: 2020-05-05.

World Health Organization (WHO) 2020. Corona virus disease (COVID-19) pandemic, WHO https://worldometer.info/coronavirus.Assesedon.Jan 28, 2021. 09:09

Worldometer, "Coronavirus Cases," Worldo meter, pp. 122, 2020, doi: 1101/2020.01.23.20018549V2. Assessed on Jan 28, 2021. 09:09 GMT

Yichi L., Bowen W., Ruiyang P., Chen Z., Yonglong Z., Zhuoxun L, Xia J. And, Bin Z. 2020. Mathematical Modelling and Epidemic Prediction of COVID-19 and Its Significance to Epidemic Prevention and Control Measures. Annals of Infectious Disease and Epidemiology 5 (1): 1052.

Yonar H., Yonar A, Tekindal M.A and, Tekindal M. 2020. Modelling and Forecasting for thenumber of cases of the COVID-19 pandemic with the Curve Estimation Models, the Box-Jenkins and Exponential Smoothing Methods. Eurasian J. Med. Oncol. 4(2):160-165.

Zhan C., Chi K., Lai Z., Hao T., and, Su J. 2020. Prediction of COVID-19 spreading profiles in South Korea, Italy and Iran by data-driven coding. medRxiv.. 